\title{
Butterfly speciation and the distribution of gene effect sizes fixed during adaptation
}

\author{
SW Baxter ${ }^{1}$, SE Johnston ${ }^{2}$ and CD Jiggins ${ }^{1}$ \\ ${ }^{1}$ The Department of Zoology, School of Biological Sciences, University of Cambridge, Cambridge, UK and ${ }^{2}$ Department of Animal \\ and Plant Sciences, University of Sheffield, Western Bank, Sheffield, UK
}

\begin{abstract}
Mimicry has had a significant historical influence as a tractable system for studying adaptation and is known to play a role in speciation. Here, we discuss recent theoretical treatment of adaptive walks to local adaptive peaks and contrast this with the adaptive landscape of mimicry. Evolution of novel Müllerian mimicry patterns almost certainly involves substitution of a major mutation to provide an initial similarity to the model, such that major gene effects are expected to an even greater degree than for other adaptive traits. The likelihood of large adaptive peak shifts in mimicry evolution may therefore promote speciation. In addition, mimicry adaptive peaks are determined by the local abundance of particular patterns and may be more fluid than the case for other traits. It will therefore be of considerable
\end{abstract}

Keywords: mimicry; lepidoptera; adaptive walk

\section{Introduction}

Adaptation is fundamental to evolutionary biology and yet the genetic basis of adaptation has not featured significantly in evolutionary theory. Until recently, there was no theoretical prediction regarding the number and effect size of genes expected to be fixed during adaptation and similarly, few empirical data are available to answer these questions. However, analysis of quantitative trait loci (QTL), facilitated by the availability of molecular markers, has led to an increasing understanding of the genetic basis of adaptive traits (Bradshaw et al., 1995; Rieseberg, 2001). This has been paralleled by the development of theory that makes predictions regarding walks through adaptive landscapes, broadly defined as a bout of adaptation involving several steps towards a local optima (Orr and Coyne, 1992; Orr, 2002). One of the major goals of this theoretical and empirical research is to determine the distribution of the gene effect sizes fixed during adaptation.

There is a close connection between speciation and adaptation, but the impact of the genetic architecture of adaptation on the probability of speciation is not clear (Schluter, 2000). Nonetheless, in some cases there is a

Correspondence: $\operatorname{Dr}$ CD Jiggins, Department of Zoology, School of Biological Sciences, University of Cambridge, Downing Street, Cambridge CB2 3EI, UK.

E-mail: c.jiggins@zoo.cam.ac.uk

Received 1 February 2008; revised 1 August 2008; accepted 5

September 2008; published online 5 November 2008 interest to test empirically the distribution of effect sizes fixed during mimicry evolution. Here, we show the feasibility of this by presenting a preliminary quantitative trait locus (QTL) analysis of Heliconius colour patterns. This shows that a number of modifier loci of different effect sizes influence forewing band morphology. We also show multiple pleiotropic effects of major Heliconius patterning loci and discuss the likelihood of multiple substitutions at the same loci in pattern evolution, which would inflate the importance of major loci in QTL analysis of the gene effect sizes. Analyses such as these have the potential to uncover the genetic architecture of both within and between species adaptive differences.

Heredity (2009) 102, 57-65; doi:10.1038/hdy.2008.109; published online 5 November 2008 similar genetic basis to changes within and between species and the kinds of adaptive changes that differentiate populations also differ between species (Naisbit et al., 2003; Lexer et al., 2005). Thus, an understanding of the genetics of adaptation will need to underlie any general understanding of speciation. Ultimately, we would like to make predictions about what kinds of adaptive landscapes and genetic systems are most likely to promote speciation. Butterfly wing colour patterns are well-studied examples of adaptations that also play a role in speciation through their pleiotropic effects on reproductive isolation. Here, we aim to address two issues. First, we discuss the existing theory of adaptive walks, and compare the adaptive landscape of mimicry with that underlying this theory, highlighting some important differences. Second, we discuss the feasibility of empirical estimation of the distribution of gene effect sizes fixed during mimicry adaptation by presenting a preliminary quantitative trait locus analysis of butterfly colour patterns in the genus Heliconius, and briefly review the current state of knowledge about the genetic architecture of mimicry.

\section{Theoretical background}

Fisher famously considered the effect size of mutations responsible for adaptation with a geometrical model, concluding that genes of very small effect were overwhelmingly more likely to be selectively advantageous (Fisher, 1930). This result was very influential and 
arguably discouraged further consideration of the distribution of gene effect sizes involved in adaptation (Orr, 2005). Subsequently, however, (Kimura, 1983) highlighted that Fisher had not considered the probability that any particular advantageous mutation would become fixed. Mutations of larger effect are likely to have a greater selective advantage, and therefore they are more likely to sweep through populations and become fixed, rather than be lost through drift. Thus, mutations of some intermediate size are actually those that are most likely to play a role in adaptation (Kimura, 1983).

More recently, Orr has considered not merely the probability of fixation of a single mutation but also the distribution of effect sizes over the course of an adaptive walk towards a local optimum. The main prediction of what now amounts to a considerable body of theory is that an exponential distribution of effect sizes is expected over a single adaptive walk (Orr, 1998, 2005). One or a few mutations are expected to account for most of the phenotypic change. Surprisingly, the predictions are similar from two very different approaches to the problem, either considering DNA sequence space or using a solely phenotypic model, and are also apparently robust to different fitness distributions and mutational models (Orr, 2005).

The testability of these predictions has nonetheless been called into question (Clarke and Arthur, 2000). In particular, the pleiotropic effects of genetic changes are likely to constrain or restrict adaptive change at certain loci, and the qualitative nature of traits such as chirality in gastropods does not easily fit with a theory based on quantitative morphological change. Nonetheless, certain forms of adaptation might eventually provide a useful empirical test of this theory. A number of studies in both animals and plants have already shown that genes of major effect play a major role in adaptation, which confirms the principle predictions in a qualitative manner (Bradshaw et al., 1995; Nachman et al., 2003; Colosimo et al., 2005a, 2005b; Steiner et al., 2007). Recently, the first quantitative tests of the theory have been carried out using sticklebacks (Albert et al., 2008). The main difficulty in testing the theory turns out to be a practical limitation on QTL mapping methods, which cannot detect the QTL of smallest effect. To minimize this, large sample sizes are needed; for example, the stickleback study used 372 individuals from a single mapping family. In addition, the theoretical prediction was altered to take methodological limitations into account by truncating the tail of smaller QTL, giving a gamma distribution, rather than the exponential distribution predicted solely on theoretical grounds. The resulting data were a reasonably good fit to the theoretical prediction, although clearly more tests are needed to confirm this initial test of the theory (Albert et al., 2008).

\section{Does the adaptive landscape of mimicry conform to the theory?}

Butterfly wing patterns and mimicry in particular are an example of an adaptation that might lead to a quantitative test of the theory. Our current understanding of butterfly wing development suggests that wing patterns involve co-option of signalling pathway modules from earlier development (Brunetti et al., 2001). Therefore, it is a reasonable hypothesis that most evolution in wing patterns occurs at wing-specific cis-regulatory elements of these genes, with minimal pleiotropic effects on other developmental functions of the same loci (Carroll et al., 2001). This hypothesis should become testable as a better understanding of wing development is reached, but if true then developmental pleiotropic constraints on wing pattern evolution may be minimal. Furthermore, mimetic butterfly colour patterns are known to contribute to speciation through pleiotropic effects on reproductive isolation. Divergent populations use colour patterns in choosing mates, and intermediate hybrid patterns are non-mimetic and selected against by predators. Thus, they are an excellent example of an easily quantified adaptation in which the links can be made between the genetics of adaptation and speciation.

The adaptive landscape of mimicry is unusual for a number of reasons. In Müllerian mimicry, where mimetic species are all distasteful, adaptive optima are defined by the local density of individuals possessing a particular pattern (Mallet and Joron, 1999a). It has earlier been argued that all the different species might be expected to converge towards an intermediate pattern (Dixey, 1909; Sheppard, 1959). Thus, the adaptive 'optimum' would be an arbitrary point in phenotype space where different species happened to converge. However, there is little empirical evidence for such coevolution between different species in empirical studies of Müllerian mimicry, and indeed, it may not be expected theoretically in nature where mimetic species typically differ in abundance and palatability (Mallet, 1999). To differentiate convergence, involving adaptive change in both species, from the one-sided evolution of rarer and/or lessprotected species towards locally common and wellprotected species, Turner coined the term advergence (Turner, 1995). If advergence is indeed more frequent than convergence, then Müllerian mimicry evolution may resemble the theoretical adaptive walk to a local optimum.

Nonetheless, the frequency-dependent nature of mimicry means that adaptive peaks are likely to be more fluid than in other systems. Predators can presumably learn any bright and memorable pattern, meaning that mimicry patterns shared among several species are essentially located at arbitrary positions in phenotype space defined by the local abundance of a particular pattern. Genetic drift in dominant mimetic species might therefore be expected to move the pattern of multiple mimetic species through phenotype space. There is some evidence for this in the clinal geographic variation seen in the expression of pattern elements. For example, the hindwing yellow band of Heliconius melpomene and Heliconius erato races varies considerably in width and shape across the range of these species. Small changes in pattern are likely to arise through genetic drift leading to the optima shifting slightly between populations. This gives rise to the observed pattern of quantitative geographic variation in phenotype. This process is similar to that proposed for the evolution of flower colour in Antirrhinum (Whibley et al., 2006), whereby hybrids show a continuous link between two very divergent natural phenotypes. It is unknown how common this 'phenotypic drift' is, but it would predict a greater number of genes of small effect influencing 
patterns than that are predicted from a simple adaptive walk model.

Furthermore, there are other ways in which the adaptive landscape of mimicry differs from that of other idealized traits. Theoretical consideration of adaptive walks has investigated only the problem of climbing local optima with peak shifts to distant adaptive peaks considered unlikely (Orr, 2005). However, it is difficult to envisage the evolution of novel Müllerian mimicry patterns without the mimic population moving across an adaptive valley to an alternate adaptive peak (a process hereafter referred to as peak shifts). Any relatively abundant and aposematic species will be recognized by local predators; hence, a shift to mimicry of a different pattern will involve crossing an adaptive valley.

The necessity for peak shifts was recognized by Nicholson (1927) and Turner, (1985) who were perhaps the first to consider the distribution of gene effect sizes fixed during mimicry evolution. Nicholson realized that an initial large step would be necessary to adapt to a novel mimicry pattern followed by an adaptive walk of smaller steps (Figure 1). The first step must confer sufficient similarity that individuals possessing the novel pattern are at least under some circumstances confused with the model. Such large mutations may be very rare, but must arise with some significant frequency over evolutionary timescales. Under this model, the 'size' of the initial step would be constrained by the distance between adaptive peaks. Thus the overall distribution of gene effect sizes would not correspond to the theoretical prediction derived from consideration of a walk to a local optimum. However, once a large mutation has become established, alleles of more minor effect will then be favoured that refine the pattern. This second step of the Nicholson model, in which the mimic pattern is refined to more closely match the model, should correspond to Orr's adaptive walk.

Batesian mimicry, in which an edible mimic converges on a distasteful model, might seem a better system for studying the evolutionary approach to an optimum, as there is no doubt about which of the players has converged on the other. The edible mimic species have unequivocally converged onto an adaptive peak defined by the distasteful model and the genetic basis of Batesian mimicry might therefore be expected to conform to the Orr model (Figure 1). However, it has been suggested on theoretical grounds that Batesian mimicry might lead to a coevolutionary struggle with the model gradually evolving to avoid the mimic (Gavrilets and Hastings, 1997). This would lead to an evolutionary walk through phenotype space with gradual change in both model and mimic and many more small effect genes than expected.

In summary, the adaptive landscape of mimicry does show some important differences from the idealized landscape described by theoretical models of adaptive walks. Adaptation through peak shifts is expected to lead to larger initial genetic changes than expected when climbing local optima, and 'phenotypic drift' might lead to more minor effect alleles than expected. Nonetheless, it will clearly be a valuable exercise to collect empirical data on the distribution of effect sizes among mimicry genes and compare this with theoretical predictions. Any deviations along the lines described earlier might give insights into the shape of the adaptive landscape of mimicry. Furthermore, it would also be useful to extend the theory to more 'unusual' scenarios such as frequency-dependent selection and perhaps generate more quantitative predictions specific to mimicry. Next, we discuss the current state of knowledge of mimicry adaptation in Heliconius in the light of this discussion.

\section{Genetics of mimicry in Heliconius}

Many Heliconius species are composed of several geographic races with divergent mimetic patterns, which offer the opportunity to study the genetic basis of mimicry adaptation. If one race is assumed to represent the ancestral pattern relative to the more derived form then crosses between such races can be used to study the evolutionary outcome of an adaptive walk. This is likely to be a reasonable assumption in most cases, given the recency of race formation in species such as $H$. melpomene (Flanagan et al., 2004). Classic crossing experiments carried out over many years have shown a key role for major Mendelian loci in controlling pattern variation (Sheppard et al., 1985). Major pattern elements are commonly expressed in an 'all-or-nothing' manner controlled by alleles at a single or few loci. Arguably,

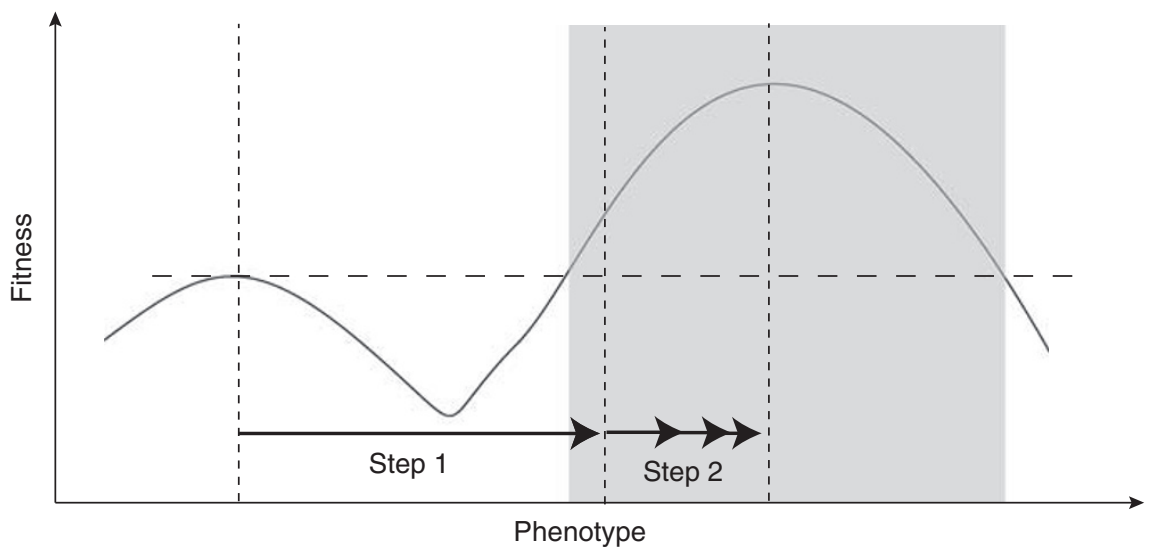

Figure 1 The 'two-step' evolution of a novel adaptation. The evolution of a novel Müllerian mimicry pattern likely involves a peak shift, as shown here, from the ancestral pattern on the left to the mimicry of a new model shown on the right by the higher fitness peak. An initial large mutational step (step 1) must confer sufficient similarity to the model to lead to some increase in fitness; in this case, it must fall within the grey box. Subsequent perfection of the pattern must involve multiple mutational steps indicated by the series of arrows in step 2 and may conform to the 'Orr model' of phenotypic evolution (Orr, 1998). 
these major gene effects correspond to the peak shifts discussed earlier (step 1 in Figure 1) and represent the initial stages of speciation, leading to colour pattern races of Heliconius that are partially reproductively isolated (Jiggins et al., 2004).

Nonetheless, major gene effects are only part of the story, as there is also considerable variation in the expression of these phenotypes in hybrid broods. This has long been recognized with most of the classical literature including discussion of 'modifier loci' that alter the expression of major pattern elements (Sheppard et al., 1985). The term 'modifier loci' implies a special class of pattern QTL, in which gene effects are epistatic with major Mendelian loci-in other words the effect of variation at the 'modifier loci' is specific to the expression of a particular major gene allele (these have sometimes been called 'special modifiers' to highlight their specificity of action (Turner, 1977)). These loci likely represent the result of adaptive walks towards local optima (step 2 in Figure 1), subsequent to fixation of a novel major effect allele.

\section{QTL analysis of modifier loci}

Here, we present a preliminary QTL analysis of 'modifier loci' affecting the expression of a major pattern element in Heliconius. The limited sample size and small number of trait variables mean that this is not intended as a quantitative test of adaptation theory, but rather as a proof of principle. We use already published molecular markers for a single pair mating of $\mathrm{F}_{1}$ hybrid offspring between $H$. m. cythera and $H$. m. melpomene, known as Brood 33 (Br33) (see Jiggins et al 2005 for details). Both races have a red forewing band, the presence of which is regulated by a dominant allele of the locus $\mathrm{HmB}$ on linkage group 18. There is a significant variation in the size and morphology of the red band with the west Ecuadorean form $H$. m. cythera showing a much narrower band as compared with the French Guianan $H . m$. melpomene. This difference is apparently adaptive as the co-mimic species, $H$. erato, shows parallel variation in the same trait. All Br33 progeny display a red forewing band that is highly variable in size and shape.

We have initially taken advantage of the lack of crossing over in female Lepidoptera to carry out a chromosome-by-chromosome analysis of this pattern variation (Figure 2; Table 1). We performed linear measurements of the $\mathrm{F}_{2}$ hybrid wings-first to adjust for size between related individuals and second to measure variability in position and size of the red forewing band. Using wing veins to determine fixed points of reference, three measurements were taken to standardize wing size from photographs of Br33 progeny wings (Figure 2 ). To quantify variation in the red $B$ band, a number of measurements were taken, and four with the highest repeatability $(>0.9)$ and large variance between individuals were chosen for subsequent analysis (Figure 2). All measurements were repeated two times per individual for both left and right wings using ImageJ software (Abramoff et al., 2004), and the average of the four measurements was used for subsequent analysis. Red band measurements were then divided by the average of the three size measurements to correct for wing size, and data were imported to MiniTab. In addition, the relative extent of the red band $\left(\mathrm{mm}^{2}\right)$ was calculated by dividing the red wing area by total wing area also using ImageJ (Table 1). After adjusting the scale, photographs were converted to eight-bit black and white images and adjusted for brightness, contrast and threshold. The option 'analyse particles' was used to generate the total wing outline plus red band outline, and then measurements were performed on colour photographs using these outlines as boundaries. For measurement protocol see http://rsb.info.nih.gov/ij/ docs/examples/index.html (area measurements and particle counting).

The measured distances and values for relative red band area were then compared with earlier generated chromosome prints (Jiggins et al., 2005), which provide genotypes for each of the 21 linkage groups of this species and follow segregation of the non-recombining female-informative chromosome. $P$-values were generated using analysis of variance with a general linear model to identify any significant association between segregation of a chromosome and the morphology of $B$.

Overall, six chromosomes were found to have a significant effect at the $P<0.005$ level at one or more of the wing measurements including an effect of sex (note that sex is determined by which sex chromosome is inherited from the mother in female-heterogametic Lepidoptera; Table 1). Interestingly, two of the chromosomes correspond to those already known to contain major wing pattern loci from earlier crosses. Linkage group 18 contains the $H m B$ locus, which controls qualitative expression of the forewing band phenotype (Joron et al., 2006). The overall size of the red forewing band was strongly influenced by this chromosome. In addition, linkage group 10 contains the locus $\mathrm{HmAc}$, which places a anvil-shaped mark on the forewing of yellow-banded races of $H$. melpomene, and is possibly homologous to HeSd, which influences many aspects of forewing band shape in $H$. erato (Joron et al., 2006). It is therefore possible that the HmAc locus is also responsible for influencing band shape in this cross, although a lack of molecular markers close to this locus currently preclude a fine-scale QTL scan of this region. In addition, sex-specific effects have been documented in earlier Heliconius crosses (Turner, 1977). These are generally revealed only in hybrid individuals and provide further evidence for adaptive modification of patterns, in this case, to reduce sexual dimorphism within mimetic populations. Finally, the analysis also reveals an effect of at least three further chromosomes, which have not been earlier documented as affecting wing pattern in Heliconius, linkage groups 2, 7 and 13 (with a conservative $P$-value of 0.005 , Table 1 ).

In particular, linkage group 13 was the only chromosome found to have a significant influence on all four of the pattern measurements and showed the highest F-values for two of the linear measurements (Table 1). We therefore carried out a QTL analysis of this chromosome using already published molecular markers (Jiggins et al., 2005). $\mathrm{F}_{2}$ regression interval mapping was carried out using QTL Express (Seaton et al., 2002) and provided strong evidence for at least one QTL at approximately $60 \mathrm{cM}$ along this chromosome influencing at least two of the linear measurements (Figure 3).

In summary, these data represent the first quantitative analysis of wing pattern variation in $H$. melpomene and provide evidence for a distribution of both large and small 'modifier' loci influencing the expression of a 
a
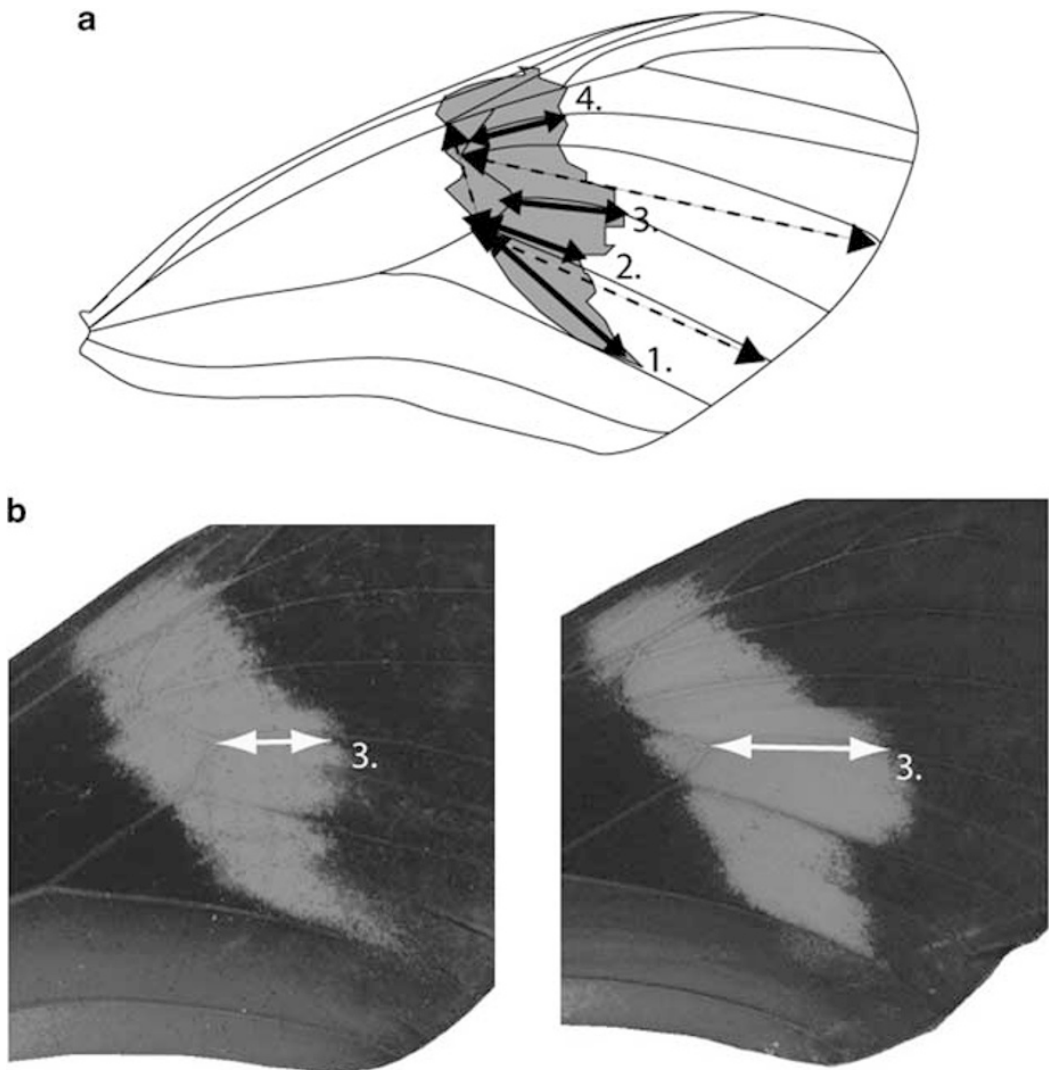

Figure 2 Linear measurements of red forewing band size. (a) Schematic diagram of H. melpomene ventral forewing, displaying veins and the red band. Dashed arrows represent measurements taken to standardize wing size. Full arrows represent four linear measurements taken to calculate band length and width. (b) Examples of ventral wings from two $F_{2}$ siblings displaying different size patterns for measurement 3 (white arrows).

Table 1 Chromosome-by-chromosome analysis of quantitative variation in the red forewing band

\begin{tabular}{|c|c|c|c|c|c|c|c|c|c|c|}
\hline \multirow[t]{3}{*}{ Linkage group } & \multicolumn{8}{|c|}{ Linear measurements } & \multicolumn{2}{|c|}{ Red band area } \\
\hline & \multicolumn{2}{|c|}{1} & \multicolumn{2}{|c|}{2} & \multicolumn{2}{|c|}{3} & \multicolumn{2}{|c|}{4} & \multirow[b]{2}{*}{$F$} & \multirow[b]{2}{*}{$P$} \\
\hline & $F$ & $P$ & $F$ & $P$ & $F$ & $P$ & $F$ & $P$ & & \\
\hline LG1 $(K)$ & 4.350 & 0.048 & 2.890 & 0.102 & 1.270 & 0.271 & 2.050 & 0.165 & 0.570 & 0.459 \\
\hline LG2 & 11.790 & 0.002 & 0.000 & 0.952 & 0.030 & 0.867 & 0.360 & 0.555 & 3.140 & 0.095 \\
\hline LG3 & 2.750 & 0.110 & 4.910 & 0.036 & 2.560 & 0.123 & 0.330 & 0.572 & 0.000 & 0.954 \\
\hline LG4 & 0.550 & 0.465 & 3.590 & 0.070 & 1.690 & 0.206 & 0.960 & 0.337 & 4.040 & 0.060 \\
\hline LG5 & 0.870 & 0.361 & 3.050 & 0.093 & 1.400 & 0.249 & 0.260 & 0.613 & 1.950 & 0.181 \\
\hline LG6 & 1.190 & 0.286 & 0.040 & 0.848 & 0.560 & 0.462 & 0.570 & 0.458 & 1.910 & 0.185 \\
\hline LG7 & 0.070 & 0.800 & 0.170 & 0.682 & 2.140 & 0.157 & 10.850 & 0.003 & 0.230 & 0.635 \\
\hline LG8 & 2.050 & 0.165 & 0.760 & 0.392 & 0.150 & 0.701 & 0.000 & 0.973 & 0.020 & 0.886 \\
\hline LG9 & 1.840 & 0.188 & 2.510 & 0.126 & 0.420 & 0.523 & 2.640 & 0.117 & 0.070 & 0.796 \\
\hline LG10 $(A c)$ & 14.870 & 0.001 & 0.350 & 0.559 & 0.990 & 0.330 & 4.330 & 0.048 & 0.160 & 0.693 \\
\hline LG11 & 5.940 & 0.023 & 1.770 & 0.196 & 1.920 & 0.179 & 3.130 & 0.090 & 3.340 & 0.085 \\
\hline LG12 & 1.920 & 0.178 & 6.680 & 0.016 & 2.940 & 0.099 & 0.050 & 0.830 & 0.550 & 0.469 \\
\hline LG13 & 5.360 & 0.029 & 9.110 & 0.006 & 16.990 & 0.000 & 25.980 & 0.000 & 1.990 & 0.176 \\
\hline LG14 & 3.310 & 0.081 & 1.140 & 0.296 & 1.860 & 0.185 & 0.000 & 0.993 & 6.540 & 0.020 \\
\hline $\operatorname{LG15}(Y b, S b$ and $N)$ & 1.000 & 0.327 & 0.330 & 0.574 & 1.360 & 0.255 & 0.200 & 0.656 & 0.000 & 0.995 \\
\hline LG16 & 0.640 & 0.433 & 0.220 & 0.646 & 1.320 & 0.262 & 2.980 & 0.097 & 3.470 & 0.080 \\
\hline LG17 & 0.720 & 0.404 & 1.190 & 0.285 & 0.910 & 0.350 & 0.260 & 0.617 & 1.310 & 0.267 \\
\hline LG18 ( $B$ and $D$ ) & 2.810 & 0.107 & 4.290 & 0.049 & 1.480 & 0.236 & 0.210 & 0.652 & 16.830 & 0.001 \\
\hline LG19 & 0.080 & 0.779 & 0.850 & 0.366 & 0.120 & 0.737 & 5.620 & 0.026 & 0.010 & 0.928 \\
\hline LG20 & 1.680 & 0.208 & 0.880 & 0.358 & 0.790 & 0.384 & 0.220 & 0.647 & 0.100 & 0.759 \\
\hline Z-chromosome & 0.000 & 1.000 & 15.440 & 0.001 & 4.670 & 0.041 & 6.160 & 0.020 & 0.000 & 0.963 \\
\hline
\end{tabular}

Abbreviations: F, F-values; GLM, general linear model; LG, linkage group; P, P-values.

Four measurements (see Figure 2) were corrected for variation in wing size and analysed in $55 \mathrm{~F}_{2}$ individuals from mapping family Br33.

F-values and $P$-values are derived from a GLM analysis using Minitab, and as all chromosomes were compared in the same model, the $P$-values are not corrected for multiple tests. 'Red band area' was calculated as red band area/total wing area for 48 of the 55 progeny. Major pattern gene names identified in an earlier study on H. melpomene are shown in brackets next to the linkage group (Joron et al., 2006). 


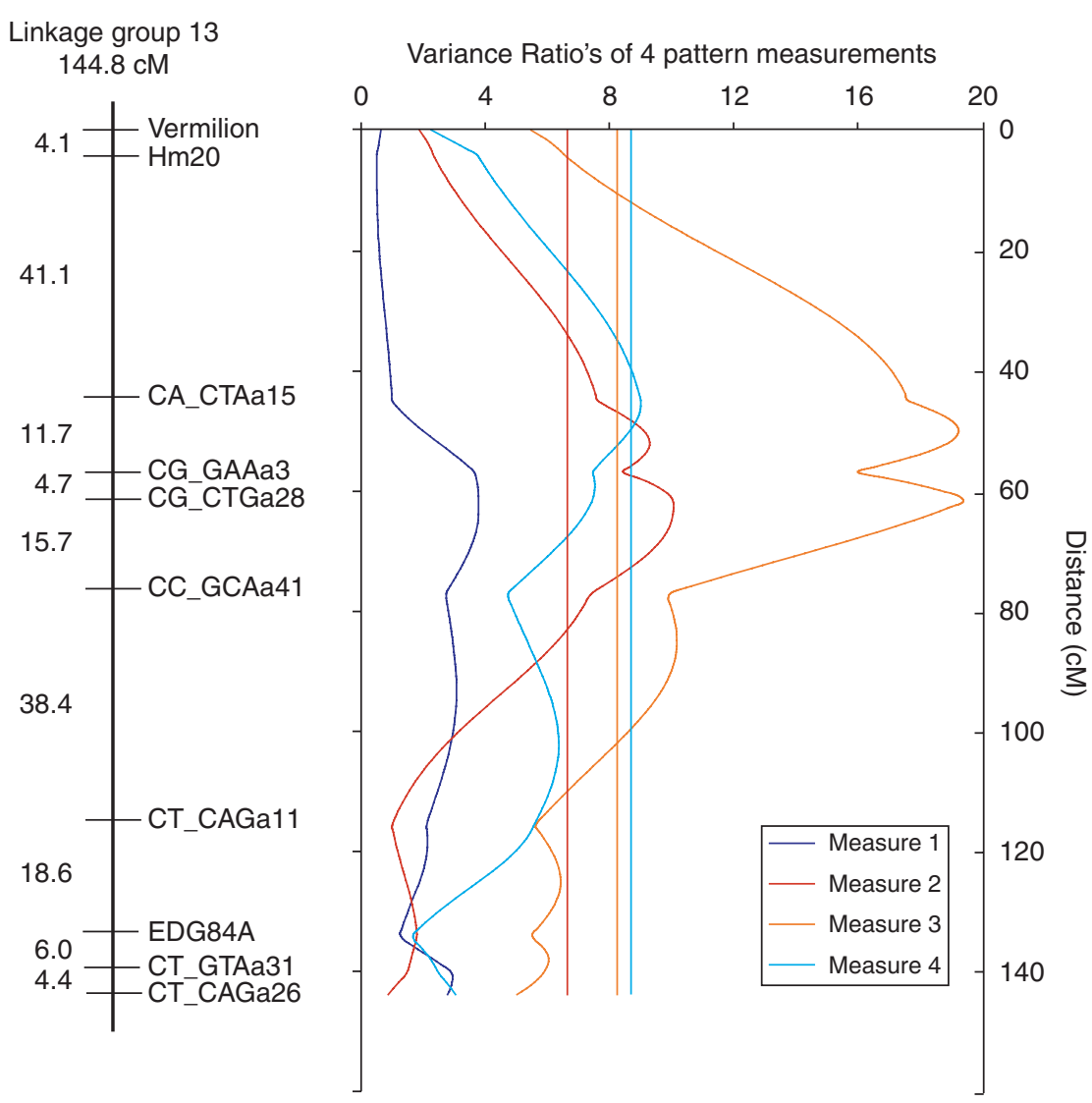

Figure $3 \mathrm{~F}_{2}$ regression interval mapping of four wing measurements. The linkage map was assembled with MapMaker 2.0 using earlier generated data (Jiggins et al., 2005), where all loci are amplified fragment length polymorphism (AFLP) markers except for one microsatellite (Hm20) and two genes (Vermilion and EDG84a). Quantitative trait locus (QTL) express was used to calculate variance ratios for the four measurements and estimate significance by whole-chromosome permutation with 1000 replicates. Significance at $P<0.01$ is shown for each measurement by coloured vertical lines, except in the case of measurement 1 that was not significantly supported.

major pattern element. We argue that these QTL correspond to the distribution of substitutions seen in step 2 of the Nicholson model (Figure 1). In the case of the red band, substitution of the novel $H m B$ allele controlling the presence or absence of the phenotype would correspond to step 1 in this process. Hence, QTL analysis directly addresses the distribution of gene effect sizes seen during mimicry adaptation, potentially allowing a comparison with theoretical predictions discussed earlier. To carry out a more robust empirical estimate of this effect size distribution will obviously require much larger sample sizes, although these are feasible in Heliconius where more than 350 individuals from replicate crosses have been used for mapping major gene loci (Baxter et al., 2008). Furthermore, crosses can also be carried out between sympatric species, offering an opportunity to compare the distribution of gene effect sizes in within versus between species crosses. This will directly address the question of whether speciation involves the same adaptive processes as adaptive evolution within species.

\section{Additional allelic effects of major loci}

An additional complication to studying the genes involved in mimicry evolution is that there is extensive evidence for multiple pleiotropic effects of major loci on different aspects of colour pattern. This means that analysis of wing patterns one trait at a time is unlikely to capture all the phenotypic effects of each QTL. An example is the unusual pattern of the east Ecuadorean form H. e. notabilis, which has two spots in the distal part of the forewing with white and pink colouration. These are largely controlled by the loci $\mathrm{HeD}$ and $\mathrm{HeSd}$ already identified in earlier crosses (Papa et al., 2008). These loci were already known to influence the forewing band colour $(\mathrm{HeD})$ and shape $(\mathrm{HeSd})$ in races with yellow or red forewing bands. However, additional loci (named Wh and Ro) had earlier been proposed to explain the segregation of the unusual forewing phenotype of H. e. notabilis (Sheppard et al., 1985). The more recent study used molecular markers to show the homology of these loci with HeD and HeSd (Papa et al., 2008).

We have also documented similar pleiotropic effects of major loci in $H$. melpomene. The mimic of $H$. e. notabilis is $H$. m. plesseni, and we have studied the genetic basis of this pattern by crossing $H$. m. plesseni with $H . m$. melpomene, following the same F2 crossing design as described for Br33. However, unlike Br33, such crosses show no clear Mendelian segregation of major pattern elements with the exception of a single colour trait. The colour of the dorsal forewing band in F2 progeny is red, but on the ventral side the band colour is either pale pink or deep orange. This likely represents the overlaying of either white or yellow ventral scales onto the deep red colour of the dorsal wing surface. In Br115, consisting 

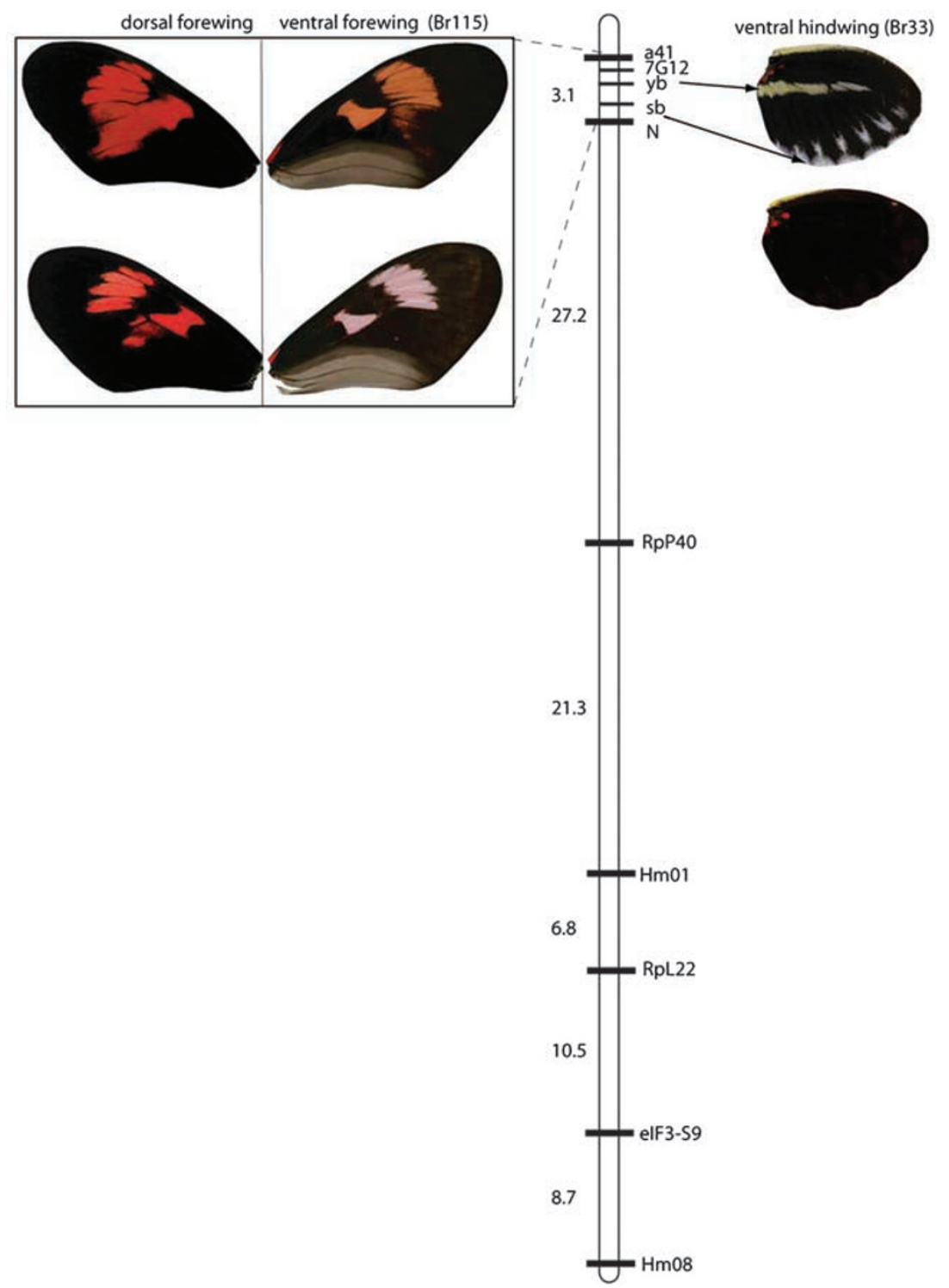

Figure 4 A forewing pink/orange ventral switch genetically maps to the same locus as a yellow hindwing bar on linkage group 15 . Genetic map adapted from Joron et al. (2006) and was constructed using 148 individuals $H$. melpomene brood Br33 segregating for $Y b$ and $S b$, with ventral hindwings showing the presence or absence of the two phenotypes. The pink/orange phenotype was mapped to LG15 using microsatellite $\mathrm{Hm} 08$ (Jiggins et al., 2005), and to the $Y b / S b$ region using marker 7G12 T7 (unpublished: primers $5^{\prime}-3^{\prime}$ forward: ATTCAATTGACGCTGTAT; reverse: GCATTGCAAAAAGTCTAA). If $\mathrm{F}_{1}$ parental genotypes are: mother $\mathrm{P}_{1} \mathrm{O}_{1}$ and father $\mathrm{P}_{2} \mathrm{O}_{2}$; the 33 ventral 'pink' $\mathrm{P}_{1} \mathrm{P}_{2} \mathrm{~F}_{2}$ individuals were homozygous for 7G12_T7 genotypes and all 87 ventral 'orange' $\mathrm{F}_{2}$ individuals $\mathrm{P}_{1} \mathrm{O}_{2}, \mathrm{P}_{2} \mathrm{O}_{1}, \mathrm{O}_{1} \mathrm{O}_{2}$ progeny were heterozygotes or homozygous for alternate 7G12_T7 alleles.

of 120 progeny, the colour segregates in a pink to orange ratio of $1: 3$ (33:87) from $\mathrm{F}_{2}$ progeny indicating simple Mendelian control with pink being the recessive phenotype.

By mapping markers developed in earlier studies, we have shown that the pink/orange switch in Br115 maps to exactly the same locus as $\mathrm{HmYb} / \mathrm{HmSb}$ in $\mathrm{Br} 33$ (Figure 4). The latter loci control the presence or absence of a yellow hindwing bar $(H m Y b)$ and hindwing margin $(\mathrm{HmSb})$ in $H$. melpomene, and are located close together $(0.3 \mathrm{cM})$ on linkage group 15 (Jiggins et al., 2005; Joron et al., 2006). Thus, the pink/orange switch is controlled by a locus likely homologous, or at least within $1 \mathrm{cM}$ of the gene $H m Y b$. This highlights the remarkable diversity of phenotypic effects controlled by these loci, the flexibility of which perhaps explains how the dramatic diversification of Heliconius wing patterns has occurred. These pleiotropic effects also mean that analysis of single traits is insufficient to capture the true distribution of gene effects. In future, it will be necessary to carry out simultaneous analysis of all phenotypic variation segregating in a single brood to capture the different effects of each QTL.

\section{Major genes and supergenes}

In addition to multiple pleiotropic effects of major loci on different aspects of pattern, now there is also considerable evidence that major pattern loci are shared between different Heliconius species. Thus, for example, the genomic region controlling the hindwing yellow band of $H$. melpomene $(H m Y b)$ is homologous to that 
responsible for whole-wing polymorphism in the related species, H. numata (Joron et al., 2006). Similarly, a gene linked to the $\mathrm{HeD}$ locus that controls red pattern elements in $H$. erato is also tightly linked to the $H m D$ and $H m B$ loci, which have similar phenotypic effects in H. melpomene (Baxter et al., 2008). Although the resolution of these comparative studies is not yet sufficient to identify specific genes responsible for pattern change, it is highly unlikely by chance that the same $1-2 \mathrm{cM}$ region would be responsible for similar patterns in different species. The candidate regions for each of these loci likely contain approximately 30-40 genes, several of which may be involved in the wing development. Thus, multiple and repeated allelic changes at the same genomic region have been responsible for complex pattern evolution.

The homology of loci between species and the multiple pleiotropic effects of single loci within species highlight a major problem inherent in using QTL studies to analyse the distribution of gene effect sizes fixed during adaptation. Multiple substitutions at the same locus appear as a single QTL but in fact result from several adaptive steps through phenotype space. The current evidence suggests that such multiple hits at a single gene in adaptive evolution might be surprisingly common. Similarly, repeated changes have been seen at the $M C 1 R$ locus in mammalian and bird colouration and at regulatory elements of the yellow locus in Drosophila wing pattern evolution (Mundy et al., 2004; Gompel et al., 2005; Prudhomme et al., 2006). In future, it may be possible to estimate the number of mutations involved in each allelic variant using sequence and functional analysis of such gene regions. In the meantime, any test of the distribution of gene effect sizes needs to consider the possibility of multiple hits and if possible analyse very recent adaptive change to minimize their likelihood.

Our crossing experiments therefore show that the same genomic regions segregate with colour pattern in within-species crosses across Heliconius. In addition, the same loci also explain species differences when related species are hybridized, and hence they are directly implicated in speciation. For example, between $H$. melpomene and $H$. cydno the $H m Y b$ and $H m B$ genes control a large proportion of the difference in colour pattern between the species (Naisbit et al., 2003), and such differences account for a large proportion of the reproductive isolation between these species (Jiggins et al., 2001). Thus, there is a continuum from colour pattern races, differing by allelic differences at one or more of these major pattern loci and partially reproductively isolated (Jiggins et al., 2004), through to sympatric and more strongly isolated species. It seems plausible that the propensity of mimetic species to large phenotypic shifts, necessitated by the adaptive landscape of mimicry, might predispose them to adaptive radiation.

The extreme case of multiple pleiotropic effects of single loci are 'supergenes' that control polymorphisms notably among Batesian mimics (Clarke and Sheppard, 1960). Supergenes might be viewed as a 'failure to speciate' with stable polymorphism representing an alternative outcome to speciation in the face of disruptive selection. The evolution of supergenes was explained by Fisher as a result of the fixation of both linked and unlinked modifier loci, which gradually perfect the pattern that is turned on by the single switch locus (Fisher, 1930). In this way, he was able to explain the evolution of major gene control of mimetic patterns under the gradualist evolutionary framework, and counter the arguments of mutationists such as Goldschmidt (1945). The reality is most probably somewhere in between the positions of Fisher and Goldschmidt with initially major mutational effects being gradually perfected (Turner, 1985). However, as recognized by Fisher, the resulting 'supergene' system is not good evidence for single major mutational step having led to a complex adaptation. Clearly, supergenes represent a highly tuned genetic system adapted to within-species polymorphism and would not be appropriate for studying the genetic basis of adaptive walks. Nonetheless, there is also a distribution of mutational effects segregating within crosses involving supergenes, and the prediction of the Fisher model would presumably be large numbers of small effect loci modifying the expression of the supergene alleles. H. numata offers a tractable system for studying this pattern and would make an interesting contrast with inter-racial and inter-specific crosses in other Heliconius species (Joron et al., 1999; Mallet and Joron, 1999b).

\section{Conclusions}

Butterfly colour patterns offer an unusually tractable system for analysing the genetic basis of adaptation and have a long history in this regard. The fact that an entire chapter of Fishers' book, The Genetical Theory of Natural Selection was devoted to mimicry testifies to its historical importance as an empirical test case for adaptation theory (Fisher, 1930). The development of molecular markers for a number of the classic mimicry species (Joron et al., 2006; Clark et al., 2008) now offers the opportunity for a renewed contribution of mimicry theory to a wider debate about the genetic basis of adaptation (Orr, 2005; Colosimo et al., 2005b; Steiner et al., 2007). There are a number of ways in which the adaptive landscape of mimicry differs from that considered in earlier models, and we have argued that further theoretical developments may be necessary to provide predictions specific to mimetic systems. Nonetheless, QTL analysis of wing patterns will allow us to determine the distribution of the gene effect sizes fixed during mimicry evolution, subject to inherent limitations of the method. Here, we have carried out a preliminary study of quantitative variation in a single wing pattern element and shown evidence for a distribution of different-sized 'modifier loci' influencing pattern expression. A more complete analysis of QTL variation in pattern will require development of methods to quantify whole-wing phenotypic variation and whole genome QTL analysis as presented here for a single chromosome. If major gene effects are significantly larger than predicted under the adaptive walk theory, this might provide evidence in support of peak shifts and the Nicholson 'two-step' theory of mimicry evolution.

\section{Acknowledgements}

We thank the University of Edinburgh project students who contributed to the QTL analysis, Susan Chapple and Christine Crossan and Mathieu Joron for discussion and 
comments on a draft of the paper. This study was funded by the BBSRC and a Royal Society fellowship to CJ.

\section{References}

Abramoff MD, Magelhaes PJ, Ram SJ (2004). Image Processing with ImageJ. Biophotonics International 11: 36-42.

Albert AYK, Sawaya S, Vines TH, Knecht AK, Miller CT, Summers BR et al. (2008). The genetics of adaptive shape shift in sticklebacks: pleiotropy and effect size. Evolution 62: 76-85.

Baxter S, Papa R, Chamberlain N, Humphray S, ffrench-Constant $\mathrm{R}$, McMillan WO et al. (2008). Parallel evolution in the genetic basis of Mullerian mimicry in Heliconius butterflies. Genetics (in press; doi:10.1534/genetics.107.082982).

Bradshaw HD, Wilbert SM, Otto KG, Schemske DW (1995). Genetic mapping of floral traits associated with reproductive isolation in monkeyflowers (Mimulus). Nature 376: 762-785.

Brunetti CR, Selegue J, Monteiro A, French V, Brakefield PM, Carroll S (2001). The generation and diversification of butterfly eyespot color patterns. Current Biology 11: 1578-1585.

Carroll S, Grenier JK, Weatherbee SD (2001). From DNA to Diversity. Blackwell: Malden, MA, USA.

Clark R, Brown SM, Collins SC, Jiggins CD, Heckel DG, Vogler A (2008). Colour pattern specification in the Mocker Swallowtail Papilio dardanus: the transcription factor invected is a candidate for the mimicry locus $H$. Proc $R$ Soc B-Biol Sci 275: 1181-1188.

Clarke B, Arthur W (2000). What constitutes a 'large' mutational change in phenotype? Evol Dev 2: 238-240.

Clarke CA, Sheppard PM (1960). The evolution of mimicry in the butterfly Papilio dardanus. Heredity 14: 163-173.

Colosimo PF, Hosemann KE, Balabhadra S, Villareal G, Dickson M, Grimwood J et al. (2005a). Widespread parallel evolution in sticklebacks by repeated fixation of Ectodyplasin alleles. Science 307: 1928-1933.

Colosimo PF, Peichel CL, Nereng KS, Blackman BK, Shapiro MD, Schluter D et al. (2005b). The genetic architecture of parallel armor plate reduction in threespine sticklebacks. PLoS Biology 2: 0635-0641.

Dixey FA (1909). On Mullerian Mimicry and Diaposematism. A reply to $\mathrm{Mr}$ GAK Marshall. Transactions of the Entomological Society of London, XVIII, pp 559-583.

Fisher RA (1930). The Genetical Theory of Natural Selection. Clarendon Press: Oxford.

Flanagan N, Tobler A, Davison A, Pybus OG, Kapan DD, Planas $S$ et al. (2004). The historical demography of Müllerian mimicry in the Neotropical Heliconius butterflies. Proc Natl Acad Sci USA 101: 9704-9709.

Gavrilets S, Hastings A (1997). Coevolutionary chase in twospecies systems with applications to mimicry. J Theoret Biol 191: $415-427$.

Goldschmidt RB (1945). Mimetic polymorphism, a controversial chapter of Darwinism. Q Rev Biol 20: 147-164; 205-230.

Gompel N, Prudhomme B, Wittkopp PJ, Kassner VA, Carroll SB (2005). Chance caught on the wing: cis-regulatory evolution and the origin of pigment patterns in Drosophila. Nature 433: 481-487.

Jiggins CD, Estrada C, Rodrigues A (2004). Mimicry and the evolution of pre-mating isolation in Heliconius melpomene. J Evol Biol 17: 680-691.

Jiggins CD, Mavarez J, Beltrán M, Johnston JS, Bermingham E (2005). A genetic map of the mimetic butterfly, Heliconius melpomene. Genetics 171: 557-570.

Jiggins CD, Naisbit RE, Coe RL, Mallet J (2001). Reproductive isolation caused by colour pattern mimicry. Nature 411: 302-305.

Joron M, Papa R, Beltrán M, Chamberlain N, Mavárez J, Baxter $S$ et al. (2006). A conserved supergene locus controls colour pattern diversity in Heliconius butterflies. PLoS Biology 4: 10.

Joron M, Wynne IR, Lamas G, Mallet J (1999). Variable selection and the coexistence of multiple mimetic forms of the butterfly Heliconius numata. Evolutionary Ecology 13: 721-754.
Kimura M (1983). The Neutral Theory of Molecular Evolution. Cambridge University Press: Cambridge.

Lexer C, Rosenthal DM, Raymond O, Donovan LA, Rieseberg LH (2005). Genetics of species differences in the wild annual sunflowers Helianthus annuus and $H$. petiolaris. Genetics 169: 2225-2239.

Mallet J (1999). Causes and consequences of a lack of coevolution in Mullerian mimicry. Evolutionary Ecology 13: 777-806.

Mallet J, Joron M (1999a). Evolution of diversity in warning color and mimicry: Polymorphisms, shifting balance, and speciation. Ann Rev Ecol Syst 30: 201-233.

Mallet J, Joron M (1999b). Evolution of diversity in warning colour and mimicry: Polymorphisms, shifting balance and speciation. Ann Rev Ecol Syst 30: 201-233.

Mundy NI, Badcock NS, Hart T, Scribner K, Janssen K, Nadeau NJ (2004). Conserved genetic basis of a quantitative plumage trait involved in mate choice. Science 303: 1870-1873.

Nachman MW, Hoekstra HE, D' Agostino SL (2003). The genetic basis of adaptive melanism in pocket mice. Proc Natl Acad Sci USA 100: 5268-5273.

Naisbit RE, Jiggins CD, Mallet J (2003). Mimicry: developmental genes that contribute to speciation. Evol Dev 5: 269-280.

Nicholson AJ (1927). A new theory of mimicry in insects. Austral Zool 5: 10-104.

Orr HA (1998). The population genetics of adaptation: The distribution of factors fixed during adaptive evolution. Evolution 52: 935-949.

Orr HA (2002). The population genetics of adaptation: The adaptation of DNA sequences. Evolution 56: 1317-1330.

Orr HA (2005). The genetic theory of adaptation: a brief history. Nat Rev Genet 6: 119-127.

Orr HA, Coyne JA (1992). The genetics of adaptation: a reassessment. Amer Nat 140: 725-742.

Papa R, Morrison CM, Walters JR, Counterman BA, Chen R, Halder G et al. (2008). Highly conserved gene order and numerous novel repetitive elements in genomic regions linked to wing pattern variation in Heliconius butterflies. BMC Genomics 9: 345.

Prudhomme B, Gompel N, Rokas A, Kassner VA, Williams TM, Yeh SD et al. (2006). Repeated morphological evolution through cis-regulatory changes in a pleiotropic gene. Nature 440: 1050-1053.

Rieseberg LH (2001). Chromosomal rearrangements and speciation. Trends Ecol Evol 16: 351-357.

Schluter D (2000). The Ecology of Adaptive Radiation. Oxford University Press: New York.

Seaton G, Haley CS, Knott SA, Kearsey M, Visscher PM (2002). QTL Express: mapping quantitative trait loci in simple and complex pedigrees. Bioinformatics 18: 339-340.

Sheppard PM (1959). Natural Selection and Heredity. Hutchinson and Co Ltd: London.

Sheppard PM, Turner JRG, Brown KS, Benson WW, Singer MC (1985). Genetics and the evolution of muellerian mimicry in Heliconius butterflies. Philos Trans $R$ Soc London Ser B 308: 433-613.

Steiner CC, Weber JN, Hoekstra HE (2007). Adaptive variation in beach mice caused by two interacting pigmentation genes. PLoS Biology 5: e219.

Turner JRG (1977). Butterfly mimicry-genetical evolution of an adaptation. In: Hecht MK, Steere WC and Wallace B (eds). Evolutionary Biology. Plenum Press: New York. Vol. 10, pp 163-206.

Turner JRG (1985). Fisher's evolutionary faith and the challenge of mimicry. Oxford Surveys in Evolutionary Biology 2: 159-196.

Turner JRG (1995). Mimicry as a model for coevolution. In: Arai R, Kato M and Doi Y (eds). Biodiversity and Evolution. National Science Museum Foundation: Tokyo, pp 131-150.

Whibley AC, Langlade NB, Andalo C, Hanna AI, Bangham A, Thebaud C et al. (2006). Evolutionary paths underlying flower color variation in Antirrhinum. Science 313: 963-966. 